\title{
Is waking electroencephalographic activity a predictor of daytime sleepiness in sleep-related breathing disorders?
}

\author{
E. Sforza*, S. Grandin*, C. Jouny*, T. Rochat", V. Ibanez*
}

Is waking electroencephalographic activity a predictor of daytime sleepiness in sleeprelated breathing disorders? E. Sforza, S. Grandin, C. Jouny, T. Rochat, V. Ibanez. (C) ERS Journals Ltd 2002.

ABSTRACT: No data are available in the literature assessing the potential use of waking electroencephalographic (EEG) activity in the detection of excessive daytime sleepiness (EDS) in patients with sleep-related breathing disorders (SRBD). The aim of this study was to evaluate whether waking EEG spectral power reflects the level of EDS in SRBD patients.

The study was performed in 48 patients in whom quantitative EEG analysis, including the alpha attenuation coefficient (AAC), was performed. Sleepiness was assessed by the Epworth Sleepiness Scale, the Stanford Sleepiness Scale, the Visual Analogue Scale and the maintenance of wakefulness test.

Although AAC and EEG spectral power tended to vary throughout the day, none of these variations correlated with EDS measures. Waking EEG measures were not different between snorers and apnoeic patients. Compared to nonsleepy patients, sleepy patients had greater theta and slow alpha powers, but the differences did not reach statistical significance. The EEG slowing was independent of hypoxaemia, severity of SRBD, or degree of sleep disruption.

The authors conclude that waking electroencephalographic measures are not sensitive enough to predict variation in alertness or to differentiate sleepy from nonsleepy sleeprelated breathing disorders patients. The degree of electrocephalographic slowing was related neither to sleep disruption nor to severity of sleep-related breathing disorders. Eur Respir J 2002; 19: 645-652.

\author{
*Sleep Laboratory, Division of \\ Neurophysiology, Dept of Psychia- \\ try and ${ }^{\#}$ Division of Pneumonology, \\ Geneva University Hospital, Geneva, \\ Switzerland. \\ Correspondence: E. Sforza \\ Unité de Neurophysiologie \\ Laboratoire de sommeil \\ Département de Psychiatrie \\ Hopital Belle Idée \\ 2 Chemin du Petit Bel Air \\ Ch 1225 Chêne Bourg \\ Gèneve \\ Switzerland \\ Fax: 41223055343 \\ E-mail: sforza-emilia@diogenes.hcuge.ch
}

Keywords: Alpha attenuation test daytime sleepiness

electroencephalography

sleep-related breathing disorders

Received: August 162001

Accepted after revision November 22 2001
A cardinal symptom of sleep-related breathing disorders (SRBD) is an increase in sleep propensity revealed by marked daytime sleepiness and reduced alertness. Many protocols have been applied in SRBD patients in order to obtain sufficiently accurate predictive models of the diurnal impairment. Surprisingly, measures of sleep loss [1] apnoea rate [2] and concomitant sleep fragmentation [3] have not shown strong influence on diurnal sleepiness. Although the failure to find consistent evidence of a relation suggests that sleepiness may be related to different factors, two important limitations of the existing research should be considered. First, the multiple sleep latency test (MSLT) [4] and the maintenance wakefulness test (MWT) [5, 6] may not adequately reveal diurnal impairment in sleepy patients and they do not strongly correlate with the subjective estimation [7]. Second, a decrease in mean sleep latency in the MSLT may occur even when the subjects rated themselves as alert, suggesting that subjective estimation measures different aspects of sleepiness. Thus, neither objective nor subjective measures of alertness currently characterize the phenomenon of sleepiness with complete accuracy.

Some recent studies suggest that various components of the spectral electroencephalographic (EEG) activity during wakefulness may help to detect changes in alertness. The awake alpha power with eyes-open increases when performance and alertness decrease [8], and the ratio of alpha power during eyes-closed versus eyes-open, i.e. the alpha attenuation coefficient (AAC) [9], falls in sleep-deprived subjects [10] and in narcoleptics [11]. Furthermore, awake alpha and theta powers are accurate indicators of sleep propensity, enhanced theta activity and decreased alpha activity $[12,13]$ found when sleepiness becomes manifest.

Therefore, it is reasonable to suggest that changes in waking alpha and theta EEG power, similar to those described earlier, could be expected in SRBD patients. If so, the degree of waking EEG changes would be an important predictor of reduced alertness and a useful measure of sleepiness. The main aim of the current study was to investigate the relationship between waking EEG activity and daytime sleepiness in a SRBD population. The prediction was that the alpha power and the AAC would be reduced, and that the theta power would be increased when the patient feels sleepy, and when he has a greater tendency to fall asleep. A second aim was to see whether the slowing in waking EEG activity could be related to the degree of sleep fragmentation and sleep loss occurring in patients with SRBD. 


\section{Method}

\section{Subjects}

Forty-eight consecutive patients, 14 females and 34 males with a mean age of $49.4 \pm 1.9 \mathrm{yr}$, a mean Body Mass Index (BMI) of $32.0 \pm 0.9 \mathrm{Kg} \cdot \mathrm{m}^{2}$ and a mean apnoea-hypopnoea index (AHI) of $34.6 \pm 4$ were included in the study. They had all been referred to the sleep laboratory for snoring, suspected obstructive sleep apnoea (OSA) and/or excessive daytime sleepiness. Exclusion criteria were: 1) previous treatment for sleep apnoea with nasal continuous positive airway pressure (nCPAP), corrective upper airway surgery or a mandibular advancement device; 2) presence of medical, neurological or psychiatric diseases that could account for sleepiness; 3 ) other sleep disorders such as narcolepsy or periodic leg movements; and 4) intake of any drug such as stimulants, hypnotics, benzodiazepines, and antidepressants, known to affect EEG power. Patients were informed that some of the collected data would be used for research purposes and they gave written informed consent.

\section{Nocturnal sleep studies}

Polysomnography included three EEGs (C3-A2, C4$\mathrm{A} 1, \mathrm{PZ}-02)$, right and left electrooculograms (EOG) and one electromyogram (EMG) of chin muscles for conventional sleep staging. Respiratory airflow was monitored with a nasal cannula connected to a pressure transducer (Protech2, Minneapolis, MN, USA), thoracic and abdominal respiratory movements with piezoelectric strain gauges, and tracheal sound by microphone. Arterial oxygen saturation $\left(\mathrm{Sa}_{\mathrm{a}} \mathrm{O}_{2}\right)$ was continuously measured with a finger oximeter.

Sleep was scored using the criteria of RECHTSCHAFFEN and KALES [14] for epochs of $20 \mathrm{~s}$ by a scorer experienced in the use of standard guidelines. As indices of sleep fragmentation, the number of arousals and awakenings as well as the number of sleep state transitions were defined.

Macroarousals (MA) were scored according to American Sleep Disorder Association (ASDA) criteria [15] as abrupt shifts in EEG frequency, irrespective of chin EMG changes during nonrapid-eye movement sleep (NREM) but associated with a concurrent EMG increase in rapid-eye movement (REM) sleep. The duration was extended to include MA lasting $>1.5 \mathrm{~s}$ and $<3$ s [16].

Respiratory events were scored using criteria proposed by the American Academy of Sleep Medicine (AASM) [17]. Hypopnoeas were defined as $\geqslant 50 \%$ reduction in airflow from the baseline value lasting at least $10 \mathrm{~s}$ and associated with $3 \%$ desaturation or an arousal. Apnoeas were defined as the absence of airflow from the nasal cannula signal lasting for $>10 \mathrm{~s}$. The AHI was established as the average number of apnoeas and hypopnoeas per hour of sleep. As indices of nocturnal hypoxaemia the mean $S_{\mathrm{a}}, \mathrm{O}_{2}$ and the minimal value recorded during sleep $\left(\mathrm{Sa}_{\mathrm{a}} \mathrm{O}_{2}, \mathrm{~min}\right)$ were considered.

For descriptive analysis, AHI cut-off points $<5$ (i.e. snorers), $\geqslant 5<15, \geqslant 15<30$, and $\geqslant 30$ (mild, moderate and severe OSA, respectively) were used according to AASM criteria [17].

\section{Daytime sleepiness evaluation}

To evaluate the level of sleepiness over the preceding few weeks, patients completed the Epworth Sleepiness Scale (ESS) on the morning after nocturnal polysomnography. The ESS is a validated questionnaire containing eight items that ask for self-reported probability of dozing in a variety of situations. The dozing probability ranges from 0 (never) to 3 (high probability). Normal values range $2-10$, scores $>10$ indicating daytime sleepiness [7].

The level of daytime sleepiness was assessed using four measures conducted every $2 \mathrm{~h}$ between 09:00 $\mathrm{h}$ and 17:00 $\mathrm{h}$ in the following order: a Stanford Sleepiness Scale (SSS), a Visual Analogue Scale (VAS), a waking EEG recording with eyes-open and eyesclosed, and a MWT.

The SSS [18] contains seven statements describing different levels of current alertness ranging from 1 "feeling alert and vital" to 7 "almost in reverie, lost struggle to remain awake". The patient has to choose the most appropriate description of his sleepiness level. The VAS [19] is a 10-cm line with the inscription "very sleepy" on the left and "very alert" on the right. Subjects were instructed to draw a vertical bar across the line at the point corresponding to their subjective feeling of alertness.

Prior to lights-out for each MWT session, an alpha attenuation test (AAT) was administered similar to that reported by others [9] but modified to ensure full wakefulness. For the AAT the patients were monitored polygraphically for $5 \mathrm{~min}$ while seated in a quiet, dimly lit ( $<50$ lux) room within $1 \mathrm{~m}$ of a wall upon which a $5 \mathrm{~cm}$ " $\mathrm{X}$ " made of red tape had been placed at eye level. Patients were instructed to relax and sit quietly, with their eyes closed, they were to image fixing their eyes on the red tape on the wall, while avoiding eye blinks, movements and sleep. One minute later, they were told to open their eyes. Each eyes-closed and eyes-open session was repeated twice.

After high and low filtering $(0.5-70 \mathrm{~Hz})$, EEG signals were digitized at a sampling rate of $256 \mathrm{~Hz}$ with a 12-bit resolution. The EEG signals during each AAT trial were inspected visually and 2-s epochs containing muscle activity, small body movements, eyelid movements and microsleep were marked as artefact and rejected from the analysis. Microsleep was defined by the presence of a 2-s theta activity and/ or prominent slow eye movements appearing simultaneously with alpha activity. Overall, $<2 \%$ of epochs scored as artefacts were identified and removed prior to the calculation of averages. Artefact-free 2-s epochs were subjected to spectral analysis with a Fast Fourier Transform (FFT) on the C3-A2 lead using a 2-s Hanning window. The absolute power values $\left(\mu \mathrm{V}^{2} \cdot \mathrm{Hz}^{-1}\right)$ of EEG components known to be sensitive to drowsiness were computed: theta $(4.5-7.5 \mathrm{~Hz})$, slow alpha $(8-10 \mathrm{~Hz})$, fast alpha $(10.5-12.5 \mathrm{~Hz})$, total alpha $(8-12.5 \mathrm{~Hz})$, and beta $(15.5-35 \mathrm{~Hz})$ power. The values obtained for each 2 -s epoch were averaged across each recording session and the mean absolute 
power was calculated for all analysed bands during the two periods with eyes-closed and eyes-open. The $\mathrm{AAC}$ was calculated within the alpha frequency range to obtain ratios of mean eyes-closed to mean eyesopen. Three AACs were defined respectively for slow alpha (S-AAC), fast alpha (F-AAC), and total alpha power (T-AAC). Finally, all spectral power values were averaged over trials to obtain a mean value of each EEG index during eyes-open and eyes-closed conditions. Recordings were analysed with the electrophysiological recording analyser software package (Phitools ß), Grenoble, France) for spectral analysis.

Objective daytime sleepiness was assessed with the MWT according to standard criteria [5]. This was performed by asking the patients to sit in a quiet, dark room and to try to stay awake for five sessions scheduled at 09:00, 11:00, 13:00, 15:00. and 17:00 h. All tests were terminated $15 \mathrm{~min}$ after sleep onset or after 40 min without sleep, and a mean seep latency was calculated as averaging the latencies to sleep for the five naps.

For analysis the patients were classified into two groups as subjectively or objectively sleepy when their ESS score was $>10$ [7] and/or the mean sleep latency at the MWT $<20 \mathrm{~min}[5]$.

\section{Statistical analyses}

Differences between AHI groups and between sleepy and nonsleepy patients were evaluated using a nonparametric test with Bonferroni correction for multiple comparison. The differences were considered significant if they had values of $\mathrm{p}<0.01$.

To assess variation over time in subjective and objective sleepiness for each patient, the results of each of these measures were transformed into the percentage of the mean calculated over the five diurnal sessions, and a two-way analysis of variance (ANOVA) for repeated measures was conducted with AHI type (snorers versus mild, moderate and severe obstructive sleep apnoea (OSA)) and groups (sleepy versus nonsleepy) as the between factor, and time (different times of testing) as the repeated measure. For comparisons that reached significance, post hoc analysis was performed by using the StudentNewman-Keuls method. Correlation analyses using the Pearson test were used to detect associations between variables. All statistical analyses were performed with the SPSS statistical software package (SPSS for Windows, 9.0, SPSS Inc., Chicago, IL, USA). Results are presented as means \pm SEM.

\section{Results}

\section{Characteristics of the patients}

Table 1 summarizes the clinical, anthropometric and nocturnal respiratory findings among patients stratified according to the AASM criteria. During the

Table 1. - Anthropometric, clinical and polysomnographic data of snorers and obstructive sleep apnoea (OSA) patients

\begin{tabular}{|c|c|c|c|c|c|}
\hline & \multicolumn{4}{|c|}{ Apnoea/Hypopnoea Index } & \multirow[b]{2}{*}{ p-valve } \\
\hline & $<5$ & $>5$ & $>15$ & $>30$ & \\
\hline Subjects n & 6 & 10 & 9 & 23 & \\
\hline Age yrs & $38.3 \pm 3.1$ & $45.1 \pm 2.7$ & $55.1 \pm 3.9$ & $51.9 \pm 3.0$ & 0.03 \\
\hline $\mathrm{BMI} \mathrm{Kg} \cdot \mathrm{m}^{-2}$ & $33.8 \pm 4.0$ & $36.1 \pm 2.2$ & $30.3 \pm 1.4$ & $30.5 \pm 0.9$ & NS \\
\hline AHI $n \cdot h^{-1}$ & $3.2 \pm 0.6$ & $10.2 \pm 1.1$ & $20.3 \pm 0.9$ & $59.0 \pm 4.0$ & 0.0001 \\
\hline Minimal $S_{\mathrm{a}, \mathrm{O}_{2}} \%$ & $86.7 \pm 2.1$ & $76.3 \pm 3.1$ & $76.0 \pm 1.9$ & $68.5 \pm 2.0$ & 0.0001 \\
\hline Stage $1 \%$ & $13.3 \pm 1.2$ & $18.4 \pm 2.0$ & $16.3 \pm 2.5$ & $24.2 \pm 2.0$ & 0.009 \\
\hline Stage $2 \%$ & $49.6 \pm 4.7$ & $51.1 \pm 3.8$ & $51.8 \pm 2.8$ & $56.5 \pm 1.7$ & NS \\
\hline Stage $3-4 \%$ & $18.3 \pm 4.1$ & $12.5 \pm 2.5$ & $11.6 \pm 2.1$ & $5.5 \pm 1.1$ & 0.003 \\
\hline Stage REM \% & $19.3 \pm 2.7$ & $18.1 \pm 1.5$ & $20.2 \pm 0.9$ & $13.7 \pm 0.9$ & 0.006 \\
\hline TST min & $443.7 \pm 30.5$ & $419.4 \pm 30.0$ & $454.1 \pm 14.8$ & $434.2 \pm 17.4$ & NS \\
\hline WASO min & $113.0 \pm 28.6$ & $139.4 \pm 27.0$ & $112.7 \pm 23.2$ & $131.0 \pm 18.7$ & NS \\
\hline SE \% & $79.8 \pm 5.3$ & $75.6 \pm 5.1$ & $80.6 \pm 3.6$ & $77.3 \pm 3.1$ & NS \\
\hline Sleep stage change $n$ & $212.0 \pm 18.3$ & $215.9 \pm 22.3$ & $224.8 \pm 19.7$ & $348.1 \pm 24.1$ & 0.001 \\
\hline Awakening $\mathrm{n}$ & $17.3 \pm 3.1$ & $21.1 \pm 5.6$ & $14.6 \pm 1.6$ & $19.2 \pm 3.0$ & NS \\
\hline Arousal n & $95.5 \pm 10.6$ & $136.2 \pm 19.8$ & $140.0 \pm 24.1$ & $268.1 \pm 27.2$ & 0.001 \\
\hline SL min & $26.4 \pm 4.4$ & $21.3 \pm 2.9$ & $23.9 \pm 4.7$ & $24.1 \pm 2.8$ & NS \\
\hline SSS & $2.1 \pm 0.3$ & $2.6 \pm 0.3$ & $2.2 \pm 0.2$ & $2.1 \pm 0.2$ & NS \\
\hline ESS & $8.5 \pm 0.6$ & $9.8 \pm 1.7$ & $10.6 \pm 1.3$ & $11.1 \pm 0.9$ & NS \\
\hline VAS & $1.8 \pm 0.6$ & $2.6 \pm 0.5$ & $2.1 \pm 0.6$ & $1.5 \pm 0.3$ & NS \\
\hline AAC & $5.9 \pm 2.6$ & $4.9 \pm 1.3$ & $6.2 \pm 1.1$ & $3.7 \pm 0.6$ & NS \\
\hline Theta power $\mu \mathrm{V}^{2} \cdot \mathrm{Hz}^{-1}$ & $5.7 \pm 1.4$ & $8.7 \pm 1.3$ & $4.7 \pm 0.4$ & $7.3 \pm 1.6$ & NS \\
\hline Slow alpha power $\mu \mathrm{V}^{2} \cdot \mathrm{Hz}^{-1}$ & $12.1 \pm 5.0$ & $21.6 \pm 6.7$ & $11.4 \pm 2.2$ & $16.7 \pm 5.4$ & NS \\
\hline Fast alpha power $\mu \mathrm{V}^{2} \cdot \mathrm{Hz}^{-1}$ & $9.6 \pm 4.63$ & $6.5 \pm 1.5$ & $6.8 \pm 1.1$ & $6.7 \pm 2.0$ & NS \\
\hline Total alpha power $\mu \mathrm{V}^{2} \cdot \mathrm{Hz}^{-1}$ & $21.8 \pm 8.6$ & $29.3 \pm 8.3$ & $19.8 \pm 3.5$ & $47.1 \pm 11.9$ & NS \\
\hline Beta power $\mu \mathrm{V}^{2} \cdot \mathrm{Hz}^{-1}$ & $11.8 \pm 4.3$ & $10.2 \pm 2.2$ & $9.8 \pm 1.6$ & $9.6 \pm 1.7$ & NS \\
\hline
\end{tabular}

Data presented as mean \pm SE unless otherwise stated. BMI: body mass index; AHI: apnoea plus hypopnoea index; $S_{a}, \mathrm{O}_{2}$ : arterial oxygen saturation; TST: total sleep time; WASO: wake after sleep onset; SE: sleep efficiency; SL: mean sleep latency in the maintenance wakefulness test; SSS: Stanford Sleepiness Scale; ESS: Epworth Sleepiness Scale; VAS: Visual Analogue Scale; AAC: alpha attenuation coefficient. ${ }^{\#}$ : determined using one Kruskall-Wallis test. 
day, the mean SSS was $2.2 \pm 0.13$ (range 1-5), the average VAS $1.9 \pm 0.2$ (range $0-6$ ) and the average sleep latency on the MWT 23.7 \pm 1.8 (range 0.40-40) min. Patients with greater apnoea frequency had higher ESS, although these were not significantly different and there were no differences in the SSS and VAS scores, and in the mean sleep latency in the MWT.

Pathological objective sleepiness indicated by a mean sleep latency $<20 \mathrm{~min}$ was present in 18 patients, while 23 patients had an ESS score $>10$. As seen in table 2 , the two types of patients did not differ in most of the polygraphic nocturnal parameters. Patients with an ESS score $>10$ significantly overestimated daytime sleepiness, with greater SSS and VAS scores present in these patients. In contrast, the two subgroups did not differ with respect to mean sleep latency at the MWT. Measures of subjective sleepiness showed no significant differences between patients with and without objective daytime sleepiness.

Variation over time in somnolence, alpha attenuation coefficient and waking electroencephalographic activity

Figure 1 illustrates the time-course of the SSS score, VAS score, sleep latencies, AAC, and EEG frequency bands for the five sessions of the entire group of patients. An ANOVA for repeated measures showed a significant time-of-day effect for sleep latencies in the MWT $(\mathrm{F}=7.96, \mathrm{p}<0.0001)$. The significant differences were between the lower levels at $11 \mathrm{~h}$ and $13 \mathrm{~h}$ and the highest value obtained at $17 \mathrm{~h}$. The daytime changes in mean sleep latency were not associated with significant variations in subjective estimations, both SSS $(\mathrm{F}=1.44)$ and VAS $(\mathrm{F}=1.49)$ showing no significant effect of time of day. ANOVA showed no significant inter-type or inter-group differences, the subjective and objective sleepiness variation being similar in all groups of patients stratified according to AHI and to presence or absence of daytime sleepiness.
Figure $1 \mathrm{~b}$ depicts the temporal evolution of total AAC (T-AAC), slow AAC (S-AAC) and fast AAC (F-AAC) for the whole group of patients. Whatever the alpha frequency considered, there was a pattern in AAC change contrary to that of sleep latency and subjective scores of sleepiness. During the morning hours the AAC decreased slightly but insignificantly from 09:00 h to $11: 00 \mathrm{~h}$, to peak again at 13:00 h. Thereafter, while mean sleep latency rose, an opposite downward trend was present in AAC, affecting all alpha frequencies. This trend was similar for snorers and OSA patients as well as for nonsleepy and sleepy patients, ANOVA showing no difference between types and groups of patients and times of testing.

Five EEG frequency bands were examined during the eyes-open and eyes-closed condition. Figure 1c shows the evolution of total alpha, theta and beta power at each eyes-open daytime session. For each frequency band (total alpha: $\mathrm{F}=11.3, \mathrm{p}=0.0001$; slow alpha: $\mathrm{F}=10.4, \mathrm{p}=0.0001$; fast alpha: $\mathrm{F}=6.1, \mathrm{p}=0.0002$; theta: $\mathrm{F}=11.3, \mathrm{p}=0.0001$; beta: $\mathrm{F}=3.8, \mathrm{p}=0.005$ ), a timedependent effect was detected with lowest activity level at 09:00 $\mathrm{h}$ and highest values between 11:00 $\mathrm{h}$ and 17:00 h. Variations between 11:00 $\mathrm{h}$ and 17:00 h were not statistically significant for any frequency band. There were no concordances between changes in EEG frequency bands and subjective and objective scores of sleepiness, with waking EEG measures quite unchanged after 09:00 h. There were no significant interactions between AHI severity (snorers versus OSA) and groups (sleepy versus nonsleepy) and times of testing for any frequency bands.

\section{Sleepiness measures, alpha attenuation coefficient and waking electroencephalographic power}

Since no circadian variations in AAC and waking EEG power were present for the whole group of patients, the data of the five sessions were pooled

Table 2. - Anthropometric, clinical and polysomnographic data of patients with and without daytime sleepiness

\begin{tabular}{|c|c|c|c|c|}
\hline & $\mathrm{ESS}<10$ & $\mathrm{ESS}>10$ & $\mathrm{SL}>20$ & $\mathrm{SL}<20$ \\
\hline Subjects $\mathrm{n}$ & 25 & 23 & 30 & 18 \\
\hline Age yrs & $49.6 \pm 3.0$ & $49.2 \pm 2.3$ & $53.8 \pm 2.4$ & $42.1 \pm 2.1 * *$ \\
\hline $\mathrm{BMI} \mathrm{Kg} \cdot \mathrm{m}^{-2}$ & $31.3 \pm 1.2$ & $32.8 \pm 1.3$ & $31.8 \pm 1.0$ & $32.4 \pm 1.7$ \\
\hline AHI $n \cdot h^{-1}$ & $31.6 \pm 5.8$ & $37.9 \pm 5.4$ & $37.1 \pm 5.2$ & $30.5 \pm 6.2$ \\
\hline Minimal $\mathrm{S}_{\mathrm{a}, \mathrm{O}_{2}} \%$ & $74.6 \pm 2.4$ & $72.9 \pm 1.8$ & $74.9 \pm 1.8$ & $72.1 \pm 2.7$ \\
\hline TTS min & $426.1 \pm 17.4$ & $446.8 \pm 13.9$ & $416.6 \pm 14.3$ & $468.5 \pm 15.7 * *$ \\
\hline WASO min & $141.1 \pm 18.5$ & $111.8 \pm 13.7$ & $147.6 \pm 15.0$ & $92.7 \pm 16.4^{* *}$ \\
\hline SE \% & $75.7 \pm 3.2$ & $80.2 \pm 2.3$ & $74.4 \pm 2.5$ & $83.7 \pm 2.8 * *$ \\
\hline Sleep stage change $n$ & $264.0 \pm 25.0$ & $298.2 \pm 19.4$ & $290.5 \pm 21.3$ & $263.7 \pm 23.9$ \\
\hline Awakening n & $17.5 \pm 2.1$ & $19.6 \pm 3.3$ & $19.4 \pm 2.3$ & $17.0 \pm 3.4$ \\
\hline Arousal n & $181.9 \pm 21.5$ & $209.3 \pm 28.5$ & $180.8 \pm 20.3$ & $218.7 \pm 32.6$ \\
\hline SL min & $26.7 \pm 2.4$ & $20.5 \pm 2.5$ & $31.4 \pm 1.4$ & $11.1 \pm 1.4^{* *}$ \\
\hline SSS & $1.9 \pm 0.1$ & $2.6 \pm 0.2^{* *}$ & $2.1 \pm 0.2$ & $2.5 \pm 0.12$ \\
\hline ESS & $7.2 \pm 0.4$ & $13.8 \pm 0.7$ & $9.8 \pm 0.7$ & $11.4 \pm 1.1$ \\
\hline VAS & $1.3 \pm 0.3$ & $2.5 \pm 0.3^{* *}$ & $1.6 \pm 0.3$ & $2.4 \pm 0.4$ \\
\hline
\end{tabular}

Data presented as mean \pm SE unless otherwise stated. SL: mean sleep latency in the maintenance wakefulness test; ESS: Epworth Sleepiness Scale; BMI: body mass index; AHI: apnoea/hypopnoea index; TST: total sleep time; WASO: wake after sleep onset; SE: sleep efficiency; SL: mean sleep latency in the maintenance wakefulness test; SSS: Stanford Sleepiness Scale; VAS: Visual Analogue Scale; AAC: alpha attenuation coefficient; **: $\mathrm{p}<0.01$ Mann-Whitney U-test. 

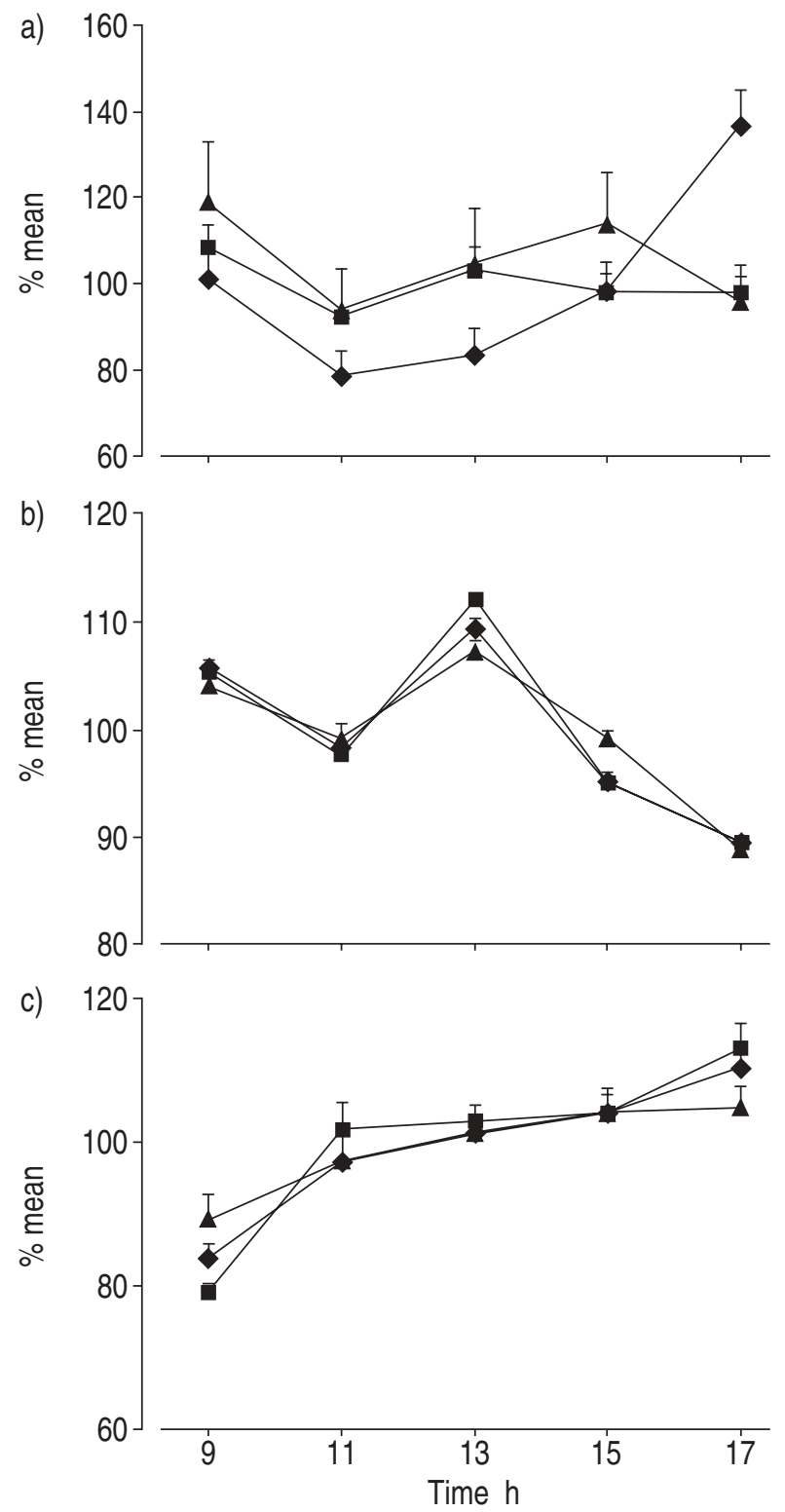

Fig. 1.-a) The time course of sleep latency as assessed by the maintenance of wakefulness test $(\bullet)$, the Stanford Sleepiness Scale ( $\boldsymbol{\square})$ and Visual Analogue Scale $(\boldsymbol{\Delta})$. b) Temporal evolution of total alpha attenuation coefficient (AAC) $(\bullet)$, slow AAC $(\boldsymbol{\square})$ and fast AAC $(\boldsymbol{\Delta})$. c) The evolution of electroencephalographic frequency bands theta $(\boldsymbol{\bullet})$, total alpha $(\boldsymbol{\square})$ and beta $(\boldsymbol{\Delta})$ power at each eyes-open daytime session. Data are presented as mean \pm SEM.

together to obtain a mean diurnal value of each sleepiness measure. Figure 2 shows the mean values of AAC and waking EEG absolute powers for patients stratified according to apnoea frequency. No significant differences in AAC and or waking EEG powers were present between snorers and apnoeic patients or among apnoeics stratified according to AHI.

Comparison between sleepy and nonsleepy patients (fig. 3) showed that the two groups did not differ in terms of waking EEG measures. Patients with an ESS $>10$ had a higher slow alpha ratio and an increase in slow alpha and theta power, but the differences did not reach statistical significance. A small decrease in

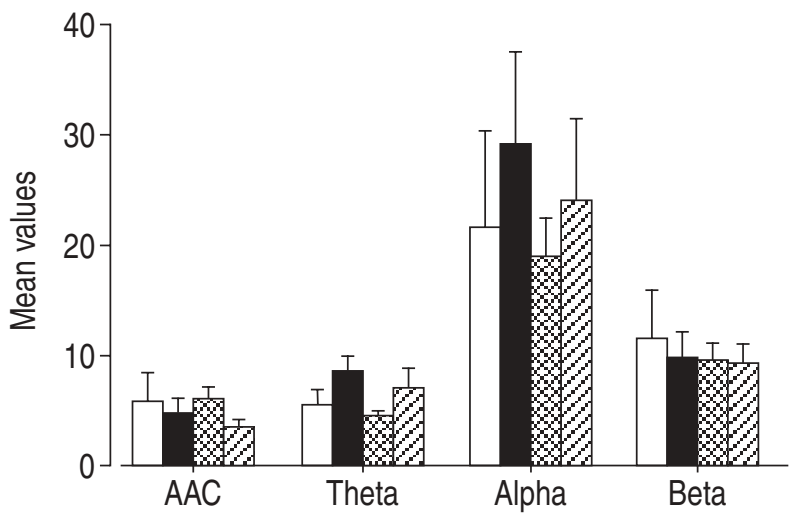

Fig. 2.-Histograms showing the average value of the alpha attenuation coefficient and awake electroencephalographic power bands for patients stratified according to apnoea-hypopnoea index (AHI). $\square$ : AHI $<5$; $\mathbf{\square}:$ AHI $>5<15$; $:$ AHI $>15<30$; $\mathbb{Z}:$ AHI $>30$.

beta EEG frequency was present in patients with objective sleepiness, but again without a significant difference.

No correlations were found between the waking EEG powers and AAC and the AHI, nocturnal hypoxaemia, and indices of sleep fragmentation.

\section{Discussion}

In this work the relationship between daytime sleepiness and waking EEG measures in a sample of patients with varying severity of sleep-related breathing disorders was investigated, testing the potential role of quantitative EEG as a predictor of reduced alertness. There were three main findings from this study. First, subjective alertness, sleep latencies and EEG frequency bands showed diurnal variations but with different time courses, EEG power variations being quite stable whether or not patients felt sleepy. Second, even though patients with sleepiness have some changes in the absolute theta and slow alpha powers, these waking EEG measures were not sensitive enough to differentiate sleepy from nonsleepy patients. Finally, the degree of EEG slowing was related neither to sleep disruption nor to severity of sleep-disordered breathing, suggesting that these factors do not contribute to waking EEG abnormalities in SRBD patients.

Although many studies have focused on sleepdisordered breathing and sleepiness, relatively few have evaluated the effect of sleep disruption on waking EEG activity in patients with SRBD, and no published data are available evaluating the potential use of waking EEG activity in the detection of daytime sleepiness in these patients. In this study the authors hypothesized that in patients with SRBD, the alpha power and the AAC would be reduced, and the theta power would be increased, when the patients feel sleepy and when sleep latency is shorter. The results of the current study do not support this speculation, quantified EEG measures showing a temporal pattern that did not parallel the trend in 

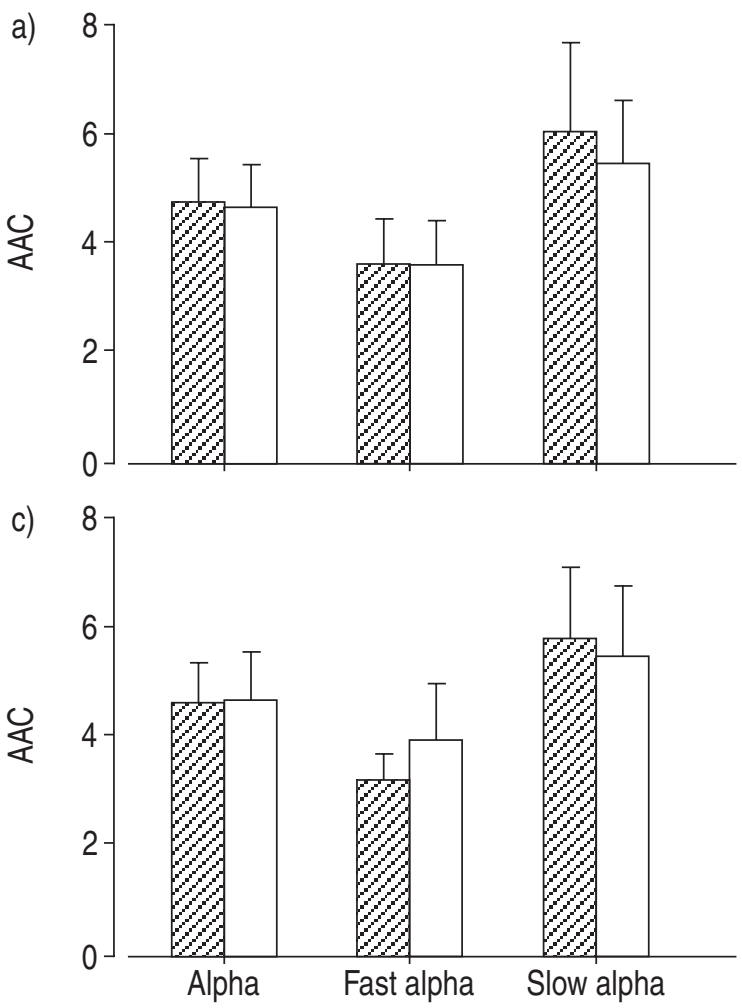
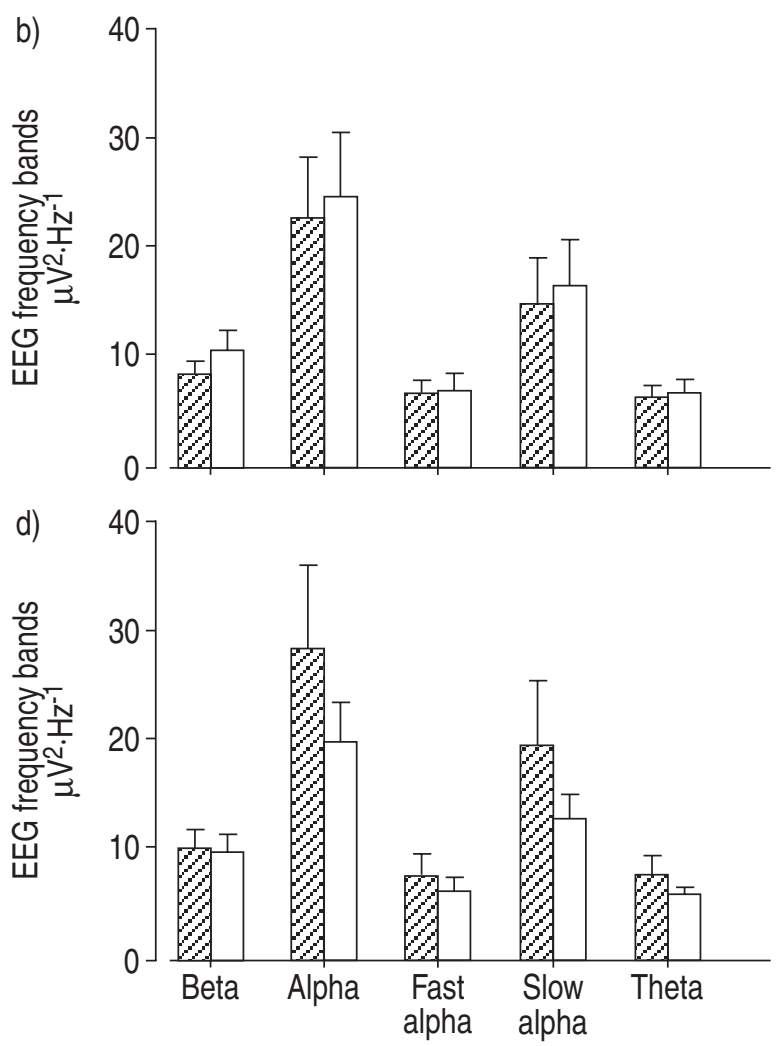

Fig. 3. - Comparison of waking electroencephalographic (EEG) measures between sleepy and nonsleepy patients, a) and b) sleepiness was assessed using the maintenance of wakefulness test (MWT). $\mathbb{Z}:$ MWT $<20 \mathrm{~min} ; \square:$ MWT $>20$ min. c) and d) using the Epworth Sleepiness Scale. $\mathbb{Z}: \mathrm{ESS}>10$; $\square$ : ESS $<10$. ACC: alpha attenuation coefficient.

objective sleepiness. The time of lowest alpha and theta power was at 09:00 $\mathrm{h}$ and thereafter alpha and theta power tended to increase or to remain stable throughout the day, independently of variation in subjective and objective sleepiness. Moreover, contrary to expectation [9], the variation over time in the AAC did not follow diurnal changes in subjective and objective sleepiness, higher values being found when sleepiness was greater. These findings seem to be in contrast with reports in the literature considering EEG activity during wakefulness in sleep-deprived subjects [20] in whom a strong association is present between sleep propensity and increase in theta activity. There are several potential explanations why waking EEG measures were not so accurate in SRBD patients. First, it is conceivable that the SSS and VAS scores are not ideal estimates of daytime sleepiness, other symptoms being more impotant markers of the diurnal impairment. This is consistent with a recent report [21] showing that fatigue, tiredness and lack of energy more than sleepiness better define the reduced alertness in patients with OSA. Second, while theta and slow alpha activity have consistently been shown to increase linearly in shiftworkers [13] and in sleep deprivation conditions [20], their changes in healthy subjects and in sleep disorder patients have not been examined. The only apparent studies in nonsleep-deprived subjects [22, 23] showed that EEG frequency bands did not follow variations in subjective and objective scores, and the relationship between theta and alpha activity and subjective alertness was lost in the whole group of subjects. These findings are consistent with these latter studies.

Comparison between sleepy and nonsleepy patients allows us to test the hypothesis of a linear relationship between degree of sleepiness and waking EEG power in SRBD patients. From previous studies it is known that the absolute powers of the whole EEG spectrum with eyes-open, are higher after total sleep deprivation, with a linear increase following the hours of prior wakefulness $[18,20]$. These changes appear to be related to the severity of sleepiness, reduced alpha power occurring when the subject rates himself as "fighting sleep", and theta power increasing when drowsiness occurs [24]. This analysis, however, failed to identify any of the waking EEG activities as a potential and specific tool to estimate excessive daytime sleepiness in SRBD patient. Although the waking power of theta and slow alpha bands was greater in patients reporting sleepiness, their sensitivity to discriminate sleepy patients was less. These findings seem to be in line with the few reports present in the literature showing that the increase in slow EEG activities during wakefulness in OSA patients is unrelated to objective sleepiness in the MSLT before [25] and after therapy [26].

A primary question that arises from these results is why some patients with SRBD and sleepiness have slowing EEG activity while awake. A first hypothesis is that sleep loss and sleep fragmentation may 
affect the restorative functions of sleep, consequently favouring slowing in the awake EEG. However, in line with previous reports $[25,26]$ this study failed to show any significant relationship between waking EEG powers and any of the markers of sleep fragmentation, confirming that these indices play little, if any, role in the occurrence of slowing of the EEG. Alternatively, it is conceivable that hypoxaemia and recurrence of apnoeas would contribute to cerebral impairment. However, no relationship between changes in waking EEG power and AHI and hypoxaemia could be demonstrated.

The lack of a close relationship between diurnal EEG measures and daytime function might have been biased by methodological factors such as study powers and absence of a control group. First, the study was designed to analyse waking EEG activity in a group of SRBD patients, excluding those in whom previous disease and medication would interfere with the spectral analysis. It is therefore possible that some of the trends in EEG measures might have been statistically significant with a larger sample size, but their clinical significance would probably be relatively small. Second, patients referred for reported apnoea and snoring, and in some cases daytime sleepiness were analysed. Since excessive sleepiness may be related to other causes, the inclusion criteria might have reduced the probability to find a relationship between EEG measures and apnoea severity. Finally, the lack of a control group limits the assessment of these measures in our patients and it seems reasonable to expect a greater correlation when controls are included. Given the known difficulty to find controls matched for BMI, in whom the physiological pattern of sleepiness and waking EEG measures could be compared to the patients, snorers were chosen as a "control group". As shown in figure 2, no differences between snorers and OSA patients were present, suggesting that the absence of controls would not have introduced a systematic bias into the results. Moreover, the diurnal trend in EEG waking measures was not different from that reported in controls [27, 28], confirming that the diurnal variation in alpha and theta powers did not correspond to that in subjective [23] and objective sleepiness [22].

In summary, notwithstanding the importance of waking electroencephalogram measurements in the evaluation of sleepiness, these results do not provide any evidence for the use of waking electroencephalogram as a sensitive means to define reduced alertness in patients with sleep-related breathing disorders. It remains to be clarified why some sleep-related breathing disorder patients had slowing in waking electroencephalogram activity and whether this abnormality reflects sleepiness. Future studies in larger samples of patients with other sleep disorders may shed further light on the mechanisms implicated in this cerebral dysfunction.

\section{References}

1. Roehrs T, Zorick F, Wittig R, Conway W, Roth T. Predictors of objective level of daytime sleepiness in patients with sleep-related breathing disorders. Chest 1989; 95: 1202-1206.

2. Kingshott RN, Engleman HM, Deary IJ, Douglas NJ. Does arousal frequency predict daytime function? Eur Respir J 1998; 12: 1264-1270.

3. Martin SE, Wraith PK, Deary IJ, Douglas NJ. The effects on non-visible sleep fragmentation on daytime function. Am J Respir Crit Care Med 1997; 155: 15961601.

4. Chervin RD, Kraemer HC, Guilleminault C. Correlates of sleep latency on the multiple sleep latency test in a clinical population. Electroencephalogr Clin Neurophysiol 1995; 95: 147-153.

5. Poceta JS, Timms RM, Jeong DO, Ho SL, Ernan MK, Mitler MM. Maintenance of wakefulness test in obstructive sleep apnea syndrome. Chest 1992; 101: 893-897.

6. Sangal RB, Thomas L, Mitler MM. Maintenance of wakefulness test and multiple sleep latency test: measurement of different abilities in patients with sleep disorders. Chest 1992; 101: 898-902.

7. Johns MW. Sensitivity and specificity of the multiple sleep latency test (MSLT), the maintenance of wakefulness test and the Epworth sleepiness scale: failure of the MSLT as a gold standard. J Sleep Res 2000; 9: 5-11.

8. Daniel RS. Alpha and theta EEG in vigilance. Percept Mot Skills 1967; 25: 697-703.

9. Stampi C, Stone P, Michimori A. The alpha attenuation test: a new quantitative method for assessing sleepiness and its relationship to the MSLT. Sleep Res 1993; 22: 115.

10. Michimori A, Stone P, Aguirre A, Stampi C. Analysis of the alpha attenuation test. Sleep Res 1994; 23: 454.

11. Alloway C, Ogilvie R, Shapiro C. The alpha attenuation test: assessing physiological sleepiness in narcolepsy. Sleep Res 1995; 24: 187.

12. Makeig S, Jung TP. Changes in alertness are a principal component of the variance in the EEG spectrum. Neuroreport 1995; 7: 213-216.

13. Akerstedt T, Torsvall L, Gillberg M. Sleepiness in shiftwork: a review with emphasis on continuous monitoring of EEG and EOG. Chronobiol Int 1987; 4: 129-140.

14. Rechtschaffen A, Kales A. A manual of standardized terminology, technique and scoring system for sleep stages of human sleep. Los Angeles Brain Information Service. Brain Information Institute, UCLA. 1968.

15. American Sleep Disorders Association. The Atlas Task Force. EEG arousals: scoring rules and examples. Sleep 1992; 15: 174-184.

16. Martin SE, Engleman HM, RN Kingshott, Douglas NJ. Microarousals in patients with sleep apnoea/ hypopnoea syndrome. J Sleep Res 1997; 6: 276-280.

17. American Academy of Sleep Medicine. Sleep-related breathing disorders in adults: recommendation for syndrome definition and measurement techniques in clinical research. Sleep 1999; 22: 667-689.

18. Hoddes E, Dement WC, Zarcone V. The development and use of the Stanford Sleepiness Scale. Psychophysiology 1972; 9: 150.
Acknowledgements. The authors would like to thank B. Adjivon, B. Bertram and A.Lalji for technical assistance. 
19. Johnson LC. Daytime sleepiness in good sleepers: measurement and correlates. In: Broughton RJ, Ogilvie RD, eds. Sleep, arousal and performance. USA. Birkhauser. 1992; 220-229.

20. Cajochen C, Brunner DP, Krauchi K, Graw P, Wirz-Justice A. Power density in theta/alpha frequencies of the waking EEG progressively increases during sustained wakefulness. Sleep 1995; 18: 890-894.

21. Chervin RD. Sleepiness, fatigue, tiredness and lack of energy in obstructive sleep apnea. Chest 2000; 118: 372-379.

22. Lafrance C, Dumont M. Diurnal variations in the waking EEG: comparison with sleep latencies and subjective alertness. J Sleep Res 2000; 9: 243-248.

23. Higuchi S, Liu Y, Yuasa T, Maeda A, Motohashi Y. Diurnal variation in alpha power density and subjective sleepiness while performing repeated vigilance tasks. Clinical Neurophysiol 2001; 112: 997-1000.

24. Akerstedt T, Gillberg M. Subjective and objective sleepiness in the active individual. Intern $J$ Neuroscience 1990; 52: 29-37.

25. Morisson F, Lavigne G, Petit D, Nielsen T, Malo J, Montplaisir J. Spectral analysis of wakefulness and REM sleep EEGs in patients with sleep apnoea syndrome. Eur Respir J 1998; 11: 1135-1140.

26. Morisson F, Decary A, Petit D, Lavigne G, Malo J, Montplaisir J. Daytime sleepiness and EEG spectral analysis in apneic patients before and after treatment with continuous positive airway pressure. Chest 2001; 119: 45-52.

27. Cacot P, Tesolin B, Sebban C. Diurnal variation of EEG power in healthy adults. Electroencephalogr Clin Neurophysiol 1995; 94: 305-312.

28. Aeschbach D, Matthews JR, Postolache TT, Jackson MA, Giesen HA, Wehr TA. Dynamics of the human EEG during prolonged wakefulness: evidence for frequency-specific circadian and homeostatic influences. Neurosci Lett 1997; 239: 121-124. 